\title{
Análisis del desarrollo social en zonas rurales aisladas empleando simulación basada en agentes
}

\author{
Yony Fernando Ceballos* \\ Omar Baqueiro Espinosa* \\ Isaac Dyner ${ }^{* * *}$
}

Recibido: 21/08/2012 • Aceptado: 06/09/2013

\begin{abstract}
Resumen
Este documento busca identificar el desarrollo humano y social en áreas rurales donde la energía juega un papel importante en la forma en la cual las personas toman decisiones y su papel en la mejora de la calidad de vida, medida como la satisfacción de las necesidades básicas de Maslow. El modelo de simulación basada en agentes (ABM) descrito, en conjunto con los resultados de simulaciones realizadas permite caracterizar y concluir respecto al impacto de la adopción de la energía en el desarrollo de una región rural. El estudio muestra que las comunidades están en capacidad de adaptarse y generar un incremento en la calidad de vida mediante el uso de la energía en la vida diaria, permitiendo mostrar que la adopción de una energía en la zona es una herramienta que posibilita el desarrollo de la región.
\end{abstract}

Palabras clave: simulación basada en agentes; desarrollo; energia; simulación social; Netlogo; zonas rurales aisladas

Instituto Tecnológico Metropolitano. Facultad de Ingenierías. Docente Auxiliar. PhD(c). Calle 54 No 30-01 Bloque N 414 - Medellín - Colombia. Telefax 4600727 ext 5615. fernandoceballos@itm.edu.co

** Leibniz institute of agricultural development in central and eastern Europe (IAMO). Investigador. PhD. TheodorLieser-Str.2 D-06120 Halle (Saale) - Alemania. Fax: +49-345-2928-399. baqueiro@iamo.de

*** Universidad Nacional de Colombia. Facultad de Minas. Docente. PhD. Carrera 80 No 65-223 - Medellín - Colombia.idyner@unal.edu.co 


\title{
Analysis of the social development in isolated rural areas through agent-based simulation
}

\begin{abstract}
The purpose of this research work is to identify the human and social development in rural areas where electric energy plays an important role in the way people make decisions, and its role in the life quality improvement, which is measured as the satisfaction of Maslow's basic needs. The described simulation model, based on agents (ABM), jointly with the results of performed simulations allow characterizing and concluding with regard to the impact of the incorporation of electric energy in the development of a rural region. The study exhibits that communities have the capability to adapt and generate an increase in the quality of life by means of the use of electric power in the day-to-day life, allowing to show that the incorporation of electric energy in the area is a tool that enables the development of the region.
\end{abstract}

Key words: agent-based simulation, development, electric energy, social simulation, NetLogo, isolated rural areas. 


\section{INTRODUCCIÓN}

En una zona rural, las personas usualmente no tienen acceso a recursos como energía eléctrica, agua potable, alcantarillado, entre otros. El Estado, como ente central, debe asegurar la disponibilidad de tales servicios, pero aparte de las razones sociales inmersas en el problema, el componente económico, es decir, la rentabilidad de la inversión no es alta, e incluso no genera retornos al Estado en muchos años. Sin embargo, si el Gobierno es democrático, este debe asegurar igualdad de condiciones de vida entre todos los individuos que pertenecen a la sociedad, haciendo que esta inversión sea necesaria.

El problema del desarrollo en diversas comunidades ha sido estudiado ampliamente y en muchas áreas de estudio [1-6]. Normalmente el desarrollo sostenible de comunidades rurales está directamente ligado a las formas en las cuales las personas que viven en una comunidad reaccionan a nuevas condiciones o se ajustan a situaciones inusuales en una forma constructiva. Diversos estudios han mostrado que la disponibilidad de energía eléctrica a comunidades rurales contribuye a su desarrollo y crecimiento [7]. No obstante, estos estudios no han tenido el impacto esperado porque son muy técnicos, empleando solo métodos econométricos o coeficientes basados en medidas tales como la línea de pobreza o miseria [8].

Antes de la inversión gubernamental en la comunidad rural, esta debe elaborar un plan de acción para lograr tener un efecto positivo en la calidad de vida de sus habitantes. Esto es porque la responsabilidad del uso y el mantenimiento de la planta de energía son asignados a las personas que viven en la región, después de un corto proceso de entrenamiento y enseñanza en el uso y mantenimiento de la tecnología. En una zona rural las personas normalmente no están bien organizadas y solo trabajan para satisfacer sus necesidades básicas de alimentación y seguridad. Haciendo una relación con la pirámide de Maslow[9] (ver figura 1), se hace posible observar sus necesidades

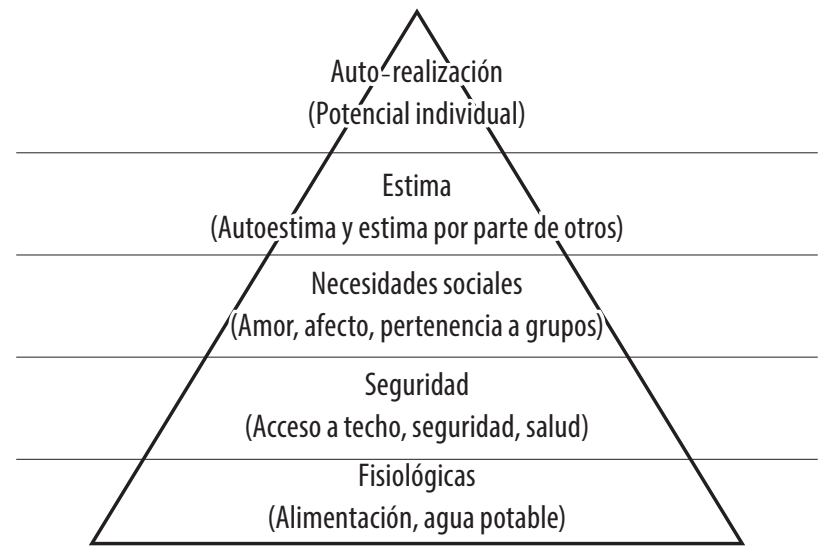

Figura 1: Pirámide de las necesidades de Maslow[19] 
relacionadas directamente con las fisiológicas (alimentación, agua), seguridad (un bajo nivel de salud, recursos, techo) y necesidades sociales (afecto, pertenencia a grupos) en la pirámide de Maslow. Todas esas personas en el interior de la comunidad normalmente satisfacen sus necesidades más apremiantes; sin embargo, pueden tener diferentes preferencias, las cuales están asociadas al tipo de individuo, y a su racionalidad. Esta hace referencia a que cada individuo al tomar decisiones, siempre está interesado en su bienestar particular antes del bienestar común. Este supuesto es muy útil en la modelación con modelos basados en agentes $(\mathrm{ABM})$ porque esta técnica está orientada a las decisiones individuales y en cómo estas decisiones pueden cambiar la evolución en sentido de desarrollo y utilización adecuada de los recursos en la comunidad. Como elementos de validación del modelo se emplearon datos aportados por expertos, los cuales pertenecen a diferentes entidades gubernamentales.

A tal fin se presenta un ABM que reproduce el efecto de la introducción de la energía (como plantas de poder) en la satisfacción de las necesidades individuales de cada grupo familiar (medido empleando la pirámide de necesidades de Maslow). La estructura del resto del documento está basada en una sección del protocolo ODD (Overview, Designing contents and Details) adaptado de Grimm et al. [10-11]. En la siguiente sección presentamos una descripción del modelo. Posteriormente, se presentan los resultados de un conjunto inicial de experimentos. Finalmente, en las dos últimas secciones, se presentan unas observaciones y conclusiones del estudio y unas posibilidades de trabajo futuro partiendo de los resultados obtenidos.

\section{CONCEPTOS DE DISEÑO}

Para esta simulación basada en agentes se emplea el protocolo ODD, que se basa en la presentación de la información necesaria para reconstruir el modelo, de una forma estructurada, como se presenta en la tabla 1.

Tabla 1. Protocolo ODD (adaptado de [10])

\begin{tabular}{|l|l|}
\hline \multirow{2}{*}{ Revisión } & Propósito \\
Conceptos de diseño & Escalas y variables de estado \\
& Revisión del proceso e itinerario \\
& Conceptos de diseño \\
Detalles & Inicialización \\
& Entradas \\
& Sub modelos \\
\hline
\end{tabular}

La revisión se analiza mediante los elementos (propósito, escalas y variables de estado y revisión del proceso e itinerario), lo cual proporciona un resumen de todo el 
proceso y estructura del modelo. Esta revisión permitiría construir, mediante un lenguaje orientado a objetos, el esqueleto principal del modelo descrito, que incluye las clases, las entidades y la secuencia de actividades a realizar por cada entidad.

El bloque de elementos de diseño no describe el modelo mismo, pero describe las partes más relevantes del mismo, para hacer posible su construcción. Este bloque, además, permite relacionar conceptos vinculados con los sistemas complejos adaptativos [12-13]. Incluye preguntas acerca de emergencia, el tipo de interacciones entre individuos considerando predicciones acerca de comportamientos futuros o por qué y cómo es la estocasticidad tenida en cuenta en el modelo.

La tercera parte a incluirse, detalles (inicialización, entradas y sub moldeos), presenta los detalles omitidos en la revisión. Los submodelos son presentados en detalle. La lógica presente en el protocolo ODD es: contextualización e información general (revisión), seguida por consideraciones más estratégicas (conceptos de diseño) y finalmente más detalles técnicos (detalles). Para una más detalla explicación del protocolo ODD remitirse a $[10,14]$.

\section{CONSTRUCCIÓN DE LA SIMULACIÓN}

Para la aproximación del modelado empleando ABM, es necesario identificar los agentes y el entorno en el cual se desenvuelven. Por lo tanto, se emplea una zona rural no interconectada al sistema central con dos o más comunidades es el universo de los agentes, las cuales pueden decidir si aceptar y utilizar la energía, permitiendo la aparición de desarrollo asociado al consumo y explotación de la misma. Solo se incluirán los 3 primeros niveles de la pirámide de Maslow (necesidades fisiológicas, de seguridad y de aceptación social) como una herramienta que permita medir en los individuos (granjas o farms) y en el área en general el nivel de desarrollo.

Propósito: El propósito de este modelo es entender cómo la introducción de tecnologías energéticas afecta el comportamiento de las personas en las zonas rurales aisladas con escaso acceso a recursos en países en vías de desarrollo. Estas áreas rurales no tienen acceso a recursos energéticos, lo cual limita la tasa de crecimiento, que se refleja en un escaso ingreso y un uso no eficiente de la tierra.

Variables de estado y escalas: Un conjunto de granjas están ubicadas aleatoriamente en cada una de las regiones. Cada granja es diferente, basada en los parámetros del cabeza de hogar (householder) y el número de personas que habitan la misma, además que es quien toma las decisiones concernientes al hogar. Este agente es racional y está representado como una granja en el modelo. El mundo es un cuadrado, en el cual las regiones son sectores de dicho cuadrado que comparten un conjunto de conexiones creadas a partir de las vecindades inmediatas de las granjas. Un decisor representa a 
toda la comunidad y solo existe uno por cada una de ellas. La simulación avanza en pasos discretos de una semana, lo cual permite simular apropiadamente una semana en una zona rural real. Para una representación posible del entorno de simulación, remitirse a la figura 2 .

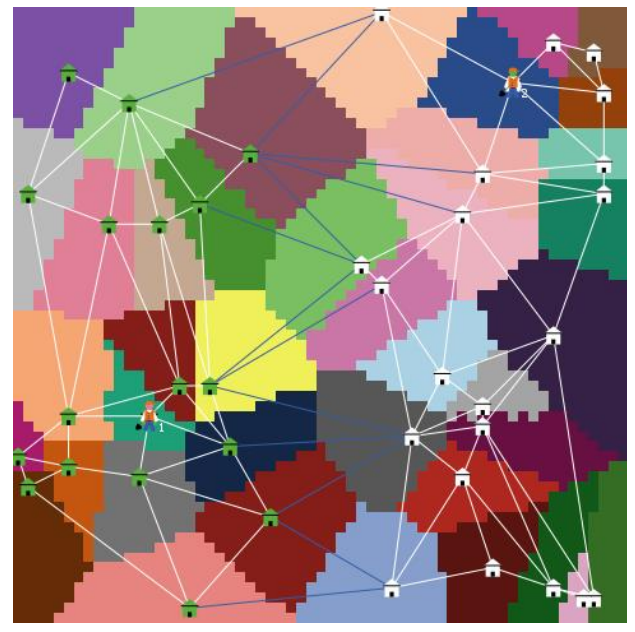

a)

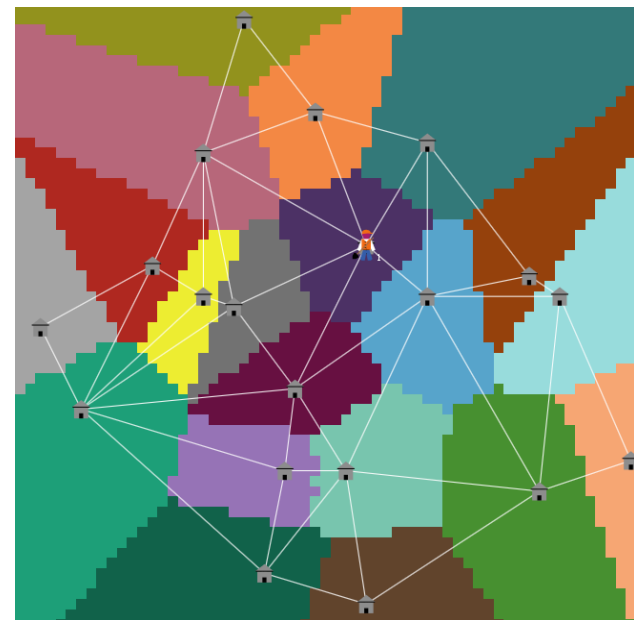

b)

Figura 2. Inicialización típica del mundo. a) Con una sola comunidad rural en la región,

b) con dos comunidades rurales en la región.

Fuente: elaboración propia

Todos los agentes deben ser inicializados con los valores para cada variable. Los valores son generados a partir de un conjunto de suposiciones hipotéticas y revisión de la literatura correspondiente (ver sección siguiente).

Revisión del proceso e itinerario. Los dos tipos de agentes que existen en el modelo son las granjas y los decisores, y están uniformemente distribuidos en la región. Para esta distribución, la única restricción es que dos agentes no pueden ubicarse en la misma posición simultáneamente. Cada cabeza de hogar posee una granja, y el decisor es también un granjero que pertenece a la comunidad, el cual, además, representa a toda la comunidad. En todo el modelo solo están presentes las granjas y los decisores, los cuales poseen las propiedades que se presentan en la figura 3. Una descripción detallada de las variables y las funciones es presentada posteriormente en la sección de submodelos. $\mathrm{Al}$ inicio de la simulación, todos los parámetros son inicializados de acuerdo con una distribución uniforme, que relaciona al tamaño de las granjas, medido en el número de parches que ocupa cada granja. Las conexiones entre granjas son creadas empleando una simple propiedad de vecindad inmediata, es decir, granjeros con granjas adyacentes son unidos. Para las granjas las variables usadas son: 


\begin{tabular}{|l|}
\hline \multicolumn{1}{|c|}{ Farm } \\
\hline Age: Integer \\
Influence: Integer \\
Energy use: Integer \\
Persons in charge: Integer \\
\hline Aging() \\
Energy adoption() \\
\hline C \\
\hline Decision-Maker \\
\hline Communitysize: Integer \\
\hline Propose energy)
\end{tabular}

Figura 3. Diagrama de clases

(Farms and Decision-Maker).

Fuente: elaboración propia

Age (A): La edad del agente cabeza de hogar (householder) medida en semanas. Los agentes mueren basados en una esperanza de vida media, calculada con un modelo de crecimiento exponencial.

Persons in charge $(P)$ : la variable personas a cargo es el tamaño de la familia que habita cada granja. Cuando un cabeza de hogar muere, se descuenta una unidad al número de personas que habitan la granja, al igual que cuando una persona nueva aparece, empleando una distribución de edades de poblaciones. Cada parámetro es cambiado basado en la edad del nuevo cabeza de hogar y los anteriores parámetros; el cambio está directamente relacionado con la pirámide de edades, presentada en la figura 4 [8].

Influence (I): esta variable es inicializada con una distribución uniforme entre $(0,0.5)$. La influencia es un número adimensional entre $(0,1)$. Solo cambia cuando el cabeza de hogar muere. Un número cercano a 1 significa que el cabeza de hogar será influenciado fácilmente al momento de adoptar la tecnología energética. En contraste, un valor cercano a 0 muestra la falta de influenciabilidad. Cada cabeza de hogar modificara sus parámetros utilizando la información recolectada de los cabezas de hogar vecinos y de los decisores. La relación entre edad $(A)$ e influenciabilidad $I$ se presenta en (1). Para más información de esta relación ver [15].

$$
3 A=260-400 I
$$




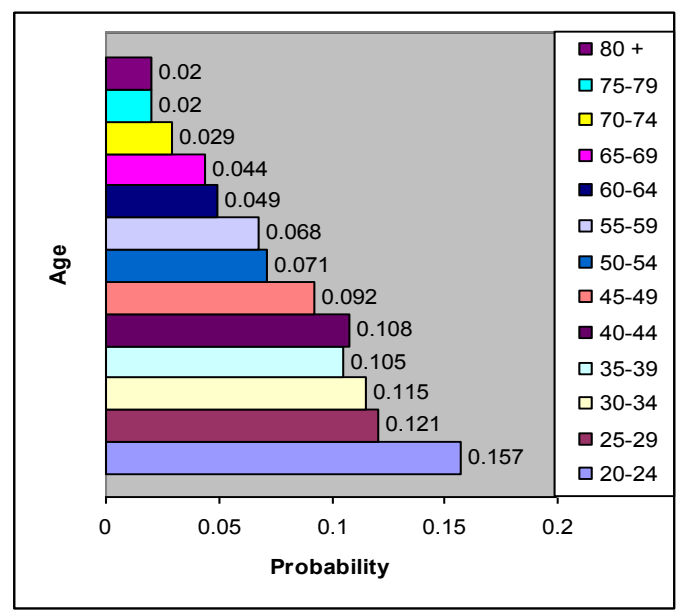

Figura 4. Distribución de las edades de los agentes del modelo.

Fuente: elaboración propia

Use coal to cook (C): las personas pertenecientes a una comunidad rural normalmente cocinan con leña. Si existe la alternativa de emplear otro medio de cocción, entonces entran las hipótesis de las libertades de Sen [16]. Cuando una comunidad tiene acceso a la energía, este atributo es inicializado aleatoriamente (con una distribución uniforme) en el rango $(0,1)$. Si no tiene acceso, se inicializa en 1 (denotando que no se posee). Para actualizarse, se emplea el procedimiento de aceptación y rechazo [17], comparando con una variable aleatoria uniforme dentro del rango. Además, este valor cambia si al menos un vecino usa energía o si directamente el decisor le convence para el empleo de la tecnología energética.

$$
C_{i+1}=C_{i}+0.2
$$

Have $T V(T V)$ : usualmente todas las personas en las zonas rurales tienen un radio o un televisor de baterías. Esta variable está relacionada con la adquisición y el acceso continuo a televisión, la cual representa la inclusión social de las personas en el país, mediante el compartir las mimas culturas e información. Esta variable solo toma 2 valores $(0,1)$. Su valor cambiará si un número aleatorio uniforme $(0,1)$ es superior a un límite de aceptación, de la misma forma que la variable "Use coal to cock" respecto a la relación (farms with TV/all farms). Después de tener valor 1 esta variable no tendrá ningún cambio. Además, la adquisición de un tv, incurre en un costo para la granja, lo cual se descontará del ingreso por paso de simulación.

Use energy in other ways $(O)$ : usualmente las personas que tienen acceso a la energía comienzan a explotarla de diferentes maneras, innovando en su uso para la 
adquisición de más ingresos o simplemente para mejorar su calidad de vida. Esta es una variable continua que comienza en cero y tiene incrementos en 0,001 , respecto a una frontera de aceptación de la influencia (I).

Farm area (FA): esta es el área de la granja, que se obtiene como la suma de todos los parches de la misma. Es un valor constante en toda la simulación; el ingreso semanal es proporcional a este valor. Por cada parche el ingreso se incrementa en 0.05 unidades monetarias (MU).

Animals (An): esta variable enumera el número de animales que la granja posee. Es inicializada de forma aleatoria con números pertenecientes a una distribución normal de media 10 y varianza 3. Es una variable que solo toma valores enteros. El beneficio por cada animal por semana está estimado en 0.05MU. Para este modelo todos los animales son considerados idénticos (las diferencias de ingresos por tipo de animal no serán consideradas.).

Energy-adopter (EA): esta variable es binaria y solo se hace cierta cuando el decisor ha aceptado emplear la tecnología energética en la comunidad.

Benefit-from-land (BfL): esta variable representa el ingreso que se obtiene por cada parche explotado en la granja. El valor inicial (7MU), se estima en función de la información obtenida con panel de expertos. Su incremento se presenta en (3).

$$
B f L_{i+1}=B f L_{i+1}+0.001
$$

Income (In): Es el resultado de la adición del ingreso por animales (0.05MU), el ingreso por área cultivada $(B f L)$, Menos el costo semanal del TV $(0.005 \mathrm{MU})$, y el costo de vida (0.1MU relacionada por el pago del uso de la energía y los alimentos que no son producidos internamente en la granja). Esta relación se presenta en (4).

$$
I n_{i+1}=B f L * F A_{i}+0.05 A n_{i}-0.005 T V_{i}-0.1
$$

House: ubicación (coordenadas cartesianas) de la casa.

Pyramid position: la ubicación de la granja en la jerarquía de Maslow. El valor de cada posición individual en la pirámide está compuesta de 3 variables independientes: necesidades fisiológicas, necesidades de seguridad y necesidades sociales (physiological needs, security y belonging needs):

Physiological needs $(P N)$ : es inicializada con valores aleatorios inferiores a $0,7 \mathrm{y}$ al mismo tiempo es calculado el valor máximo percibido por el cabeza de hogar, como se muestra en la ecuación (5).

$$
\operatorname{Max} P N=P N+\text { random }(0.3)
$$


Security needs ( $S N)$ : es inicializada con valores aleatorios inferiores a $0,5 \mathrm{y}$ al mismo tiempo es calculado el valor máximo percibido por el cabeza de hogar, como se muestra en la ecuación (6).

Max $S N=S N+$ random $(0.5)$

Belonging needs ( $B N)$ : es inicializada con valores aleatorios inferiores a 0,2 y al mismo tiempo es calculado el valor máximo percibido por el cabeza de hogar, como se muestra en la ecuación (7).

$$
\operatorname{Max} B N=B N+\text { random }(0.7)
$$

Si la granja pertenece a una comunidad que ha decidido adoptar la energía, todos los valores de la pirámide de Maslow cambian, como se presenta en la ecuación (8).

$$
P_{i+1}=P i+\beta\left(C_{i}+O_{i}+T V_{i}\right) *(\text { Income / 120) }
$$

Donde $\mathrm{P}$ es cada necesidad y $\beta$ es 0.01 para $\mathrm{PN}$ y 0.001 para el resto de necesidades; además, el efecto de ingreso es proporcional al incremento sobre el ingreso mínimo en una comunidad ideal que posee 25 granjas.

Para los decisores:

Age (Adm): La misma de los granjeros.

Beliefs: Un promedio de todas las variables, (excepto por $I$ y global position).

Households (H): El número de granjas que el decisor representa.

Influence (Idm): Este valor está compuesto por dos elementos: a) La influencia promedio de cada granja en la región y b) La idea que el decisor tiene de la energía: usualmente relacionada con la influencia o alguna política (estatal), y el aprendizaje en el uso de la energía. Esta persona representa qué tan rápido la región acepta la energía como un recurso. También pertenece al rango (0-1). Esta variable es el promedio de las dos últimas entradas y solo cambia si el agente muere. El modelo inicia con una pregunta del Gobierno central al decisor, acerca de la implementación dela tecnología energética en su comunidad. Esto solo ocurre cada año (es decir, cada 52 pasos en el modelo). Si el decisor acepta la implementación de la tecnología, todos los granjeros de la región se volverán candidatos a adoptar la tecnología energética. Cada semana, todos los granjeros adyacentes al decisor son cuestionados acerca de incluir o no la tecnología en su vida diaria. Si un granjero acepta esta influencia, todos los cambios en el uso de la tecnología ocurren, relacionado con uso de una TV, cambio del carbón gradualmente por energía eléctrica para cocer los alimentos y la compra de aparatos eléctricos que le ayudarán en diversas tareas. Cada decisor tiene su propio nivel de influencia, pero él debe representar la comunidad; por lo tanto, la comunidad puede cambiar el valor que tiene el decisor respecto a su influencia. 
Propose energy: La interacción entre el exterior es realizada por el decisor, de aceptar o no la tecnología. Esto ocurre cada año, y para cambiar este valor, un número aleatorio uniforme es generado y comparado con el valor de la variable $I d m$. Si este valor es aceptado, se procede con los cambios antes descritos.

Energy adoption: en este procedimiento si una granja tiene $E A$ con un valor verdadero y si la influencia de los vecinos surte efecto, el granjero pregunta inmediatamente si los vecinos tienen cambios en los valores de las variables (TV, $C, O$ ), y de ahí, cambia el valor de cada una de estas variables en proporción a cada granjero vecino (en promedio).

Normalmente todas las comunidades rurales en zonas rurales aisladas sin energía son muy pequeñas, lo cual hace muy costosa una inversión del Estado en electricidad para las mismas, dado el número de personas beneficiadas. En distribución inicial, puede observarse la distribución de las granjas en la comunidad y cuáles de ellas pertenecen a una comunidad rural, aparte de las líneas que representan las relaciones de vecindad inmediatas. Las granjas de color verde pertenecen a una comunidad que no fue seleccionada para acceder a la energía; las demás granjas son candidatas a la adopción de la tecnología; el color amarillo representa que la granja adoptó la tecnología hace menos de un año; naranja representa que hace más de un año, y rojo que lleva 5 años o más con la energía. La ubicación del decisor es aleatoria, pero pertenece a la comunidad y se ubica en una de las granjas.

Para la obtención de los resultados de la sección siguiente, se realizaron 400 ejecuciones del modelo descrito, empleando diferentes parámetros de probabilidades de adopción de la energía, en conjunto con la verificación del comportamiento emergente del modelo en cuanto a la evolución positiva de la satisfacción de necesidades de Maslow.

\section{RESULTADOS}

El modelo antes descrito ha sido implementado en Netlogo [18]. Los resultados son los relacionados con una comunidad que elige emplear la energía propuesta por entes exógenos al sistema. Estas primeras simulaciones son experimentales, y su objetivo principal es entender la evolución de las necesidades de la jerarquía de Maslow, en relación con la adopción de la tecnología energética. En el modelo, la variable benefit-from-land incrementa por el mejor uso y tecnificación de la agricultura, por la posibilidad de adquirir mejor información en los medios de como explotar adecuadamente la tierra de la región en cuestión. El comportamiento de las comunidades, sin la inclusión de la tecnología energética, en términos de necesidades de Maslow, mantiene una tendencia estable consistente. Sin embargo, con la inclusión de la tecnología de la energía y la comunicación entre los agentes (granjas), un aumento en la satisfacción se observa desde el momento en que la gente de la comunidad empieza a utilizar la tecnología. 
Como se muestra en la figura 5, con la inclusión de la energía en la comunidad la satisfacción de las necesidades fisiológicas crece, porque toda la gente quiere resolver esta necesidad en primer lugar, de acuerdo con la jerarquía de Maslow, pero al mismo tiempo, hay una mejora en las demás necesidades. Esto se debe a todos los habitantes de la región tiene diferentes prioridades, pero siempre la búsqueda de una mejor manera de vivir. El aumento en el valor respecto a la cobertura de las necesidades fisiológicas puede resultar en una mejor y mayor variedad de la ingesta nutricional, la posibilidad de conservar los alimentos durante más tiempo, y el uso de diferentes herramientas para la cocina, basadas en la electricidad. Además, las necesidades de seguridad pueden aumentar ligeramente debido a la posibilidad de utilizar la iluminación con energía eléctrica, para tener un mayor sentido de control sobre su granja.

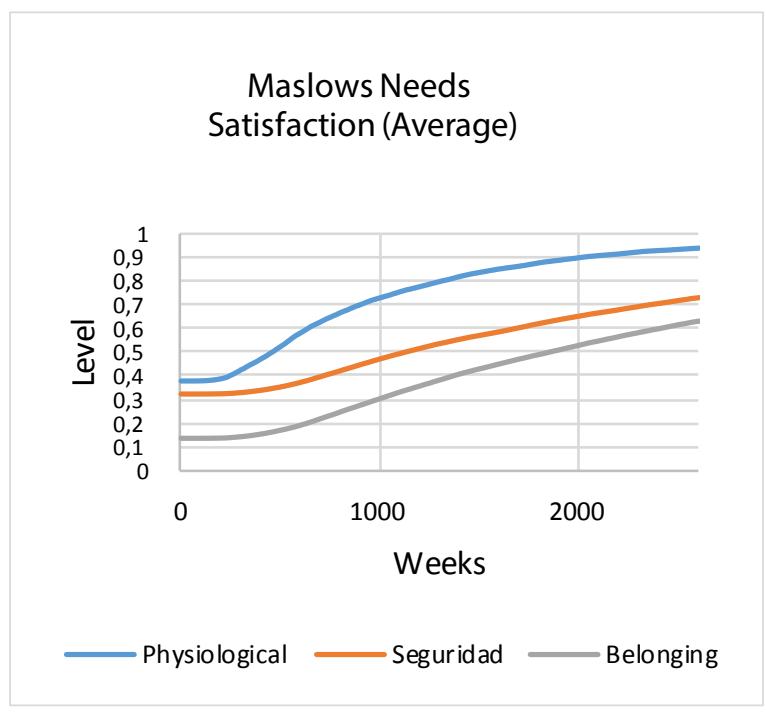

Figura 5. Satisfacción de las necesidades de la jerarquía de Maslow.

Fuente: elaboración propia

En términos de las necesidades de pertenencia a grupos, el empleo de la energía permite diferentes procesos de aprendizaje, los cuales pueden ser para las amas de casa en diferentes momentos del día, dada la dotación de energía en las escuelas y centros de salud, la posibilidad de aprender las diferentes técnicas de producción e incluso a permitir los procesos de alfabetización en la comunidad.

\section{CONCLUSIONES}

La pobreza, que ha sido discutida por las diversas autoridades, desde el punto de vista académico y social, está directamente relacionada con la calidad de vida que permite 
el pleno desarrollo en términos de necesidades básicas y adecuadas relaciones sociales. En el modelo, la pobreza se clasifica como un conjunto de factores que se analizan desde una perspectiva global, vinculando el desarrollo de la comunidad. Ya que todos ellos tienen una tendencia de crecimiento, se puede asegurar que la energía, basada en el modelo anterior, contribuye a un mejor desarrollo.

La conceptualización del problema desde el punto de vista de los componentes externos e internos implica necesariamente que las comunidades deben desarrollar el conocimiento por sus propios medios, si tienen la capacidad tecnológica como fuente de recursos para el desarrollo sostenible. La gestión de las capacidades es la de acumular conocimiento a través del aprendizaje, que incluye el intercambio de fuentes internas y externas para hacer presente la sinergia entre las partes.

\section{TRABAJO FUTURO}

Como trabajo futuro se sugiere la inclusión de un mayor número de comunidades, ya que en la situación descrita no se tienen en cuenta los posibles efectos de la elección de una comunidad rural, entre otras posibilidades. Es necesario mejorar la caracterización de las granjas para la construcción de la jerarquía. Además, no todos los datos utilizados para la inicialización de los resultados de la simulación presentada se basan en datos de una región real. Por lo tanto, es necesario mejorar la inicialización mediante la adquisición de datos que permitan su representación de una región en particular.

\section{REFERENCIAS}

[1] S. Shortall, "Are rural development programs socially inclusive? Social inclusion, civic engagement, participation, and social capital: Exploring the differences", Journal of Rural Studies, vol. 24, n. ${ }^{\circ}$, pp. 450-457, Oct. 2008.

[2] H. Soesastro, "Energy Development under Regional Autonomy: Distributions, Poverty Alleviation, Subsidies and Corporate Social Responsibilities", Economics, n. ${ }^{\circ}$ November, pp. 23-24, 2004.

[3] D. M. Kammen and C. Kirubi, "Poverty, energy, and resource use in developing countries: focus on Africa", Annals of the New York Academy of Sciences, vol. 1136, pp. 348-57, Jan. 2008.

[4] R. E. R. Lopez, G. Anriquez, S. Gulati, G. Anríquez, and R. E. López, "Structural change and sustainable development", Journal of Environmental Economics and Management, vol. 53, n. 3 , pp. 307-322, May 2007.

[5] I. Ozturk, "A literature survey on energy-growth nexus", Energy Policy, vol. 38, n. ${ }^{\circ}$ 1, pp. 340-349, Jan. 2010.

[6] V. Modi, S. McDade, D. Lallement, and J. Saghir, "Energy services for the Millennium Development Goals", Energy services for the Millennium Development Goals, 2005. 
[7] BID, "Estrategia para la reducción de la pobreza rural”, Washington, DC, 1998.

[8] B. Borroto, N. Borroto, and M. Vázquez, "Alternativas energéticas para el desarrollo de asentamientos rurales ambientalmente sostenibles”, Energía, pp. 71-77, 1998.

[9] W. Wu, "The Relationship between Incentives to Learn and Maslow's Hierarchy of Needs", Physics Procedia, vol. 24, pp. 1335-1342, Jan. 2012.

[10] V. Grimm, U. Berger, F. Bastiansen, S. Eliassen, V. Ginot, J. Giske, J. Goss-Custard, T. Grand, S. K. S. Heinz, G. Huse, and others, "A standard protocol for describing individual-based and agent-based models", Ecological Modelling, vol. 198, n. ${ }^{\circ}$ 1-2, pp. 115-126, Sep. 2006.

[11] B. Müller, F. Bohn, G. Dreßler, J. Groeneveld, C. Klassert, R. Martin, M. Schlüter, J. Schulze, H. Weise, and N. Schwarz, "Describing human decisions in agent-based models-ODD + D, an extension of the ODD protocol”, Environmental Modelling \& Software, vol. 48, pp. 37-48, Oct. 2013.

[12] M. Wood and S. DeLoach, "An overview of the multiagent systems engineering methodology", Agent-Oriented Software Engineering, pp. 1-53, 2001.

[13] S. Railsback, "Concepts from complex adaptive systems as a framework for individual-based modelling”, Ecological modelling, vol. 139, pp. 47-62, 2001.

[14] J. G. Polhill, D. Parker, D. Brown, and V. Grimm, "Using the ODD protocol for comparing three agent-based social simulation models of land use change", Model to Model, pp. 15-16, 2007.

[15] M. Morris and V. Venkatesh, "Age differences in technology adoption decisiones: inplications for a changing work force”, Personnel psycology, vol. 53, pp. 375-403, 2000.

[16] A. Sen, Development as freedom. Alfred A. Knopf, New York, 1999.

[17] I. Dyner, G. Peña, and S. Arango, Modelamiento para sistemas socioeconómicos y naturales, 1st ed. Medellín: Universidad Nacional de Colombia, 2008, p. 246.

[18] U. Wilensky, "NetLogo: Center for Connected Learning Comp.-Based Modeling”, Evanston, IL: Northwestern Univ., 1999.

[19] A. H. Maslow, "A theory of human motivation", Psychological Review, vol. 50, n. ${ }^{\circ}$, pp. 370396, 1943. 